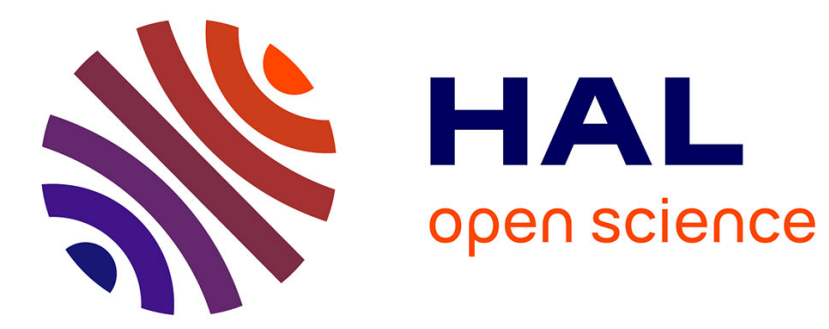

\title{
L'École aujourd'hui
}

Fabienne Serina-Karsky

\section{To cite this version:}

Fabienne Serina-Karsky. L'École aujourd'hui. Revue de l'enfance et de l'adolescence RAFEF-GRAPE, 2010, L'école autrement, 80-81, pp.61-68. 10.3917/lett.080.0061 . hal-01356845

\section{HAL Id: hal-01356845 https://hal.science/hal-01356845}

Submitted on 26 Aug 2016

HAL is a multi-disciplinary open access archive for the deposit and dissemination of scientific research documents, whether they are published or not. The documents may come from teaching and research institutions in France or abroad, or from public or private research centers.
L'archive ouverte pluridisciplinaire HAL, est destinée au dépôt et à la diffusion de documents scientifiques de niveau recherche, publiés ou non, émanant des établissements d'enseignement et de recherche français ou étrangers, des laboratoires publics ou privés. 


\section{L'ÉCOLE AUJOURD'HUI}

Fabienne Serina-Karsky

\section{ERES | «La lettre de l'enfance et de l'adolescence »}

2010/2 n 80-81| pages 61 à 68

ISSN 1146-061X

ISBN 9782749212708

Article disponible en ligne à l'adresse :

http://www.cairn.info/revue-lettre-de-l-enfance-et-de-ladolescence-2010-2-page-61.htm

\section{Pour citer cet article :}

Fabienne Serina-Karsky, "L'École aujourd'hui », La lettre de l'enfance et de l'adolescence 2010/2 ( $\left.\mathrm{n}^{\circ} 80-81\right)$, p. 61-68.

DOI 10.3917/lett.080.0061

Distribution électronique Cairn.info pour ERES.

(C) ERES. Tous droits réservés pour tous pays.

La reproduction ou représentation de cet article, notamment par photocopie, n'est autorisée que dans les limites des conditions générales d'utilisation du site ou, le cas échéant, des conditions générales de la licence souscrite par votre établissement. Toute autre reproduction ou représentation, en tout ou partie, sous quelque forme et de quelque manière que ce soit, est interdite sauf accord préalable et écrit de l'éditeur, en dehors des cas prévus par la législation en vigueur en France. Il est précisé que son stockage dans une base de données est également interdit. 


\title{
L'École aujourd'hui School for today
}

\author{
Fabienne Serina-Karsky
}

Depuis quand les enfants ont-ils leur place à l'école ? Si Charlemagne a eu cette idée folle d'inventer l'école, si Jules Ferry l'a rendue laïque, gratuite et obligatoire, l'École aujourd'hui - School for today - a pour sa part décidé d'y recevoir les enfants, avec leurs paroles, leurs rires, leurs colères, et... leurs parents aussi. Des enfants épanouis, des parents impliqués, derrière cette vision idyllique se cache le travail d'une équipe enseignante motivée par le désir d'apprendre à apprendre à des enfants accueillis à l'école dans leur globalité.

\section{Une histoire récente}

Cette petite école parisienne s'est créée en 1975 à l'initiative d'enseignantes ${ }^{1}$ bilingues à la recherche d'une école différente au sein de laquelle les enfants pourraient s'y épanouir, bientôt rejointes par un parent ${ }^{2}$ d'une école nouvelle rencontrée à Vincennes sur les bancs de l'université, dans cette nouvelle discipline qu'étaient les sciences de l'éducation. Le ton est donné dès le départ : le seul moyen de promouvoir un projet d'école original, fondé ici sur une nouvelle conception de la construction de la personnalité et une nouvelle approche de l'acquisition des connaissances ${ }^{3}$, étant de créer son propre établissement, l'école adopte une structure privée, les maris contribuent au financement lorsqu'ils le peuvent, les salaires des enseignantes attendront... deux ans ! Sous contrat simple avec l'État depuis 1977, l'école reste libre d'adapter ses pratiques pédagogiques tout en étant soumise aux mêmes obligations de programme que l'école publique.

Fabienne Serina-Karsky, doctorante en sciences de l'éducation à Paris 8, prépare une thèse intitulée La conception de l'enfant dans l'Éducation nouvelle, de la théorie à la pratique, sous la direction d'Antoine Savoye. 1. En septembre 1975, l'équipe est composée de Celie Cabane, Odile Duval, Anne Favier, Marie-Claire Franck, Claire Garçon, Sabine Michon, rejointes l'année suivante par Danielle Perrin.

2. Pierrette Kohen, qui restera à l'école vingt-sept ans, est présidente de l'association.

3. Piaget, Vygotski, Wallon et Carl Rogers sont les référents théoriques cités par Marie-Claire Franck dans le numéro spécial 30 ans du journal de l'école, Arc-en-ciel, 2005-2006. 
Regroupant pour l'année scolaire 2009-2010 cent quarante enfants répartis dans deux classes de maternelle et cinq d'élémentaire, et une équipe pédagogique gestionnaire de l'école (les neuf professeurs des écoles ${ }^{4}$, deux professeurs d'anglais anglophones ${ }^{5}$, une éducatrice de jeunes enfants ${ }^{6}$, et un enseignant chargé du soutien ${ }^{7}$, gèrent collégialement le quotidien de l'école tant sur les plans pédagogique qu'administratif et financier), l'école reste fidèle à ses principes premiers que sont l'ouverture sur le monde extérieur, avec notamment l'apprentissage de l'anglais dès la maternelle, et sa filiation à l'Éducation nouvelle, qui se traduit par une évolution constante de ses pratiques et une recherche permanente, qu'elle partage avec les autres écoles membres de l'ANEN ${ }^{8}$ lors de réunions régulières.

Quels sont les outils et pratiques pédagogiques que l'équipe de l'École aujourd'hui met en place pour " permettre aux enfants d'être les acteurs de leur formation en développant leur autonomie et en leur donnant la possibilité d'intervenir dans l'organisation et la gestion de leur travail », projet d'école élaboré lors des réunions bi-hebdomadaires de l'équipe pédagogique mais aussi lors des réunions et des conseils trimestriels parents-enseignants, la coéducation étant l'un des points forts de l'école?

\section{Accueillir l'enfant dans sa globalité}

L'école s'appuie sur les grands principes de l'Éducation nouvelle, que prônent au diapason, malgré leurs différences, John Dewey et Maria Montessori, lorsqu'ils affirment que « l'enfant est le point de départ, le centre, le but ${ }^{9}$ » et que « la véritable éducation nouvelle consiste à aller tout d'abord à la découverte de l'enfant et à réaliser sa libération ${ }^{10} »$. Cela se traduit au quotidien par la mise en place d'instances de parole et la gestion des règles communes, à travers des outils empruntés à Freinet et à la pédagogie institutionnelle : la causette et le conseil de classe, mais aussi les assemblées d'enfants et les conseils d'école.

La causette du matin permet tout d'abord de faire le lien entre la maison et l'école. Les enfants y expriment leurs soucis quotidiens, selon un rituel à respecter : l'un d'eux est désigné président de la séance et donne la parole à celui qui la demande. La parole de l'enfant s'exprime ici librement, l'enseignant s'abstenant d'intervenir, l'important n'étant pas la façon dont s'exprime l'enfant mais le sens qu'il y met. Il peut s'agir aussi bien de la présentation du doudou en maternelle, d'un livre ou d'un jeu, que de la prochaine compétition de sport ou de l'actualité pour les plus grands, mais toujours dans l'écoute et le respect de la parole de chacun.

\footnotetext{
4. Jean-Luc Bedat, Véronique Béranger, Françoise Blais, Alain Buchheit, Vanessa Mélior, Nathalie Perrin, Cécile Primot, Séverine Quesniaux, Valérie Sirat.

5. Judith Klein et Katy Renard.

6. Christelle Pauchant-Gras.

7. John Coppola.

8. L'Association nationale pour le développement de l'Éducation nouvelle, en plus de l'École aujourd'hui, regroupe en région parisienne l'École nouvelle d'Antony, l'école Emilie-Brandt à Levallois, l'école de la Rize et celle du Chapoly à Lyon, l'école de la Prairie à Toulouse.

9. Dewey, dans L'école et l'enfant p. 95-96, cité par M.-A. Bloch, Philosophie de l'éducation nouvelle, Paris, $3^{\mathrm{e}}$ éd. 1973, 218 p., p. 33.

10. Maria Montessori, L’enfant, Paris, Desclée de Brouwer, 2004, 205 p., p. 93.
} 
À travers le conseil, qui a lieu chaque vendredi, les enfants gèrent la vie de la classe. Les sujets à aborder, inscrits dans le cahier du conseil pendant la semaine, sont débattus. Ils concernent aussi bien la gestion des conflits entre les enfants que les propositions de projets pour la classe. Le projet de classe des CM2 d'élaborer un parcours de motricité pour les $\mathrm{CP}$ a ainsi pris forme ici, de même que la décision d'aller tous ensemble à la librairie choisir un livre pour la classe sur proposition d'un enfant de maternelle. Cette instance permet également aux enfants de relativiser les conflits et d'établir des priorités, il n'est en effet pas rare qu'une altercation dans la cour de récréation, se retrouvant inscrite au conseil suivant, n'y soit finalement pas débattue, l'inscription dans le cahier et la temporisation ayant suffi à dédramatiser la situation.

Lorsque le conseil ne suffit pas à régler les conflits, les enfants ont la possibilité d'avoir recours à la médiation par les pairs. Deux enfants de chaque classe élémentaire, formés à la médiation par les enseignants, se réunissent avec les enfants concernés et cherchent avec eux des solutions. Si cela ne fonctionne pas toujours, les enfants ont néanmoins la possibilité de s'exprimer et de chercher par eux-mêmes les solutions qui leur conviennent, les enseignants étant là pour intervenir lorsque ces institutions ne suffisent pas et lorsque le problème est trop lourd pour être géré par des enfants.

Quatre fois dans l'année les délégués enfants de chaque classe se réunissent pour un conseil d'école (autour d'un déjeuner !) et débattent des questions soulevées dans chaque classe, avant de rapporter à leurs camarades les décisions prises, par exemple l'élaboration de nouvelles règles pour un jeu de cour. D'autres règles, qui concernent l'école entière, sont établies en début d'année scolaire par le conseil des enfants et des enseignants. Il s'agit de règles de sécurité (ne pas courir dans les escaliers, ne pas enjamber la passerelle...), de respect des lieux (ne pas courir en chaussures dans le préau, ni dans la classe) mais aussi de respect de l'autre (ne pas siffler en travaillant, attendre que la parole soit donnée lors des causettes et conseils...). Ces règles sont complétées dans chaque classe et adaptées à l'âge des enfants.

Les enfants sont aussi respectés dans leurs rythmes de vie et d'apprentissage. Au quotidien, cela se traduit par l'alternance de séquences de travail en groupe et de travail individuel, établie sur une semaine de cinq jours, comme le préconisent les recherches sur l'aménagement du temps scolaire (Fotinos, Testu, 1996), et non sur la semaine de quatre jours imposée par l'Éducation nationale.

\section{Des enfants acteurs de leurs apprentissages}

Pour être acteurs de leurs apprentissages, les enfants ont besoin d'y mettre du sens. Selon Claparède, " la vraie pédagogie consiste à n'exercer une activité chez l'enfant qu'autant que celui-ci en ressent le besoin naturel ${ }^{11}{ }^{\prime}$. Il faut donc observer et écouter l'enfant avant de lui proposer une activité d'apprentissage. Ainsi les enfants commencent-ils, par exemple, par réaliser le modelage de leur mâchoire, puis l'empreinte de leurs dents, qu'ils vont compter et nommer (et apprendre à brosser au passage), avant de les comparer avec celles de différents animaux, ce qui

11. Claparède, dans Psychologie de l'enfant et psychologie expérimentale, 4 éd., p. 208, cité par M.-A. Bloch,

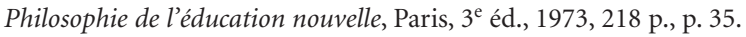


permettra d'aborder les différences d'alimentation entre les carnivores, herbivores et omnivores. Comme le prône Ovide Decroly, c'est en partant de ses centres d'intérêt que l'enfant devient acteur de sa formation. Une place importante est donc réservée aux projets personnels et exposés, qui permettent à l'enfant d'approfondir et de partager ses centres d'intérêt.

Le choix des outils pédagogiques s'appuie sur les théories de l'Éducation nouvelle ${ }^{12}$. La rencontre de l'école avec Caleb Gattegno ${ }^{13}$, pour qui l'action pédagogique de l'enseignant dans la classe doit prendre en compte, simultanément et à chaque instant, l'apprenant et la spécificité de la matière enseignée, de façon à provoquer la prise de conscience nécessaire à l'apprentissage, permet d'aborder l'apprentissage des fondamentaux par certains des outils qu'il a développés. Ainsi l'apprentissage de la lecture avec le matériel de la lecture en couleurs ${ }^{14}$, conçu pour rendre visibles les opérations de base de la lecture, comprend un ensemble d'outils mêlant chaque son de la langue française à une couleur, et permet à l'élève de se forger une pratique concrète de la lecture d'une manière ludique, enrichie par l'apport coopératif des découvertes des uns et des autres. Dans la même lignée, l'apprentissage de la grammaire se fait par les tableaux mis au point par Maurice Laurent, grâce auxquels l'élève passe de la grammaire implicite à la grammaire explicite par prises de conscience progressives. Les réglettes Cuisenaire ${ }^{15}$, présentes dans les classes dès la première année de maternelle, permettent là encore aux élèves, grâce à la représentation mentale qu'ils ont développée par leur manipulation ludique, d'aborder l'apprentissage des mathématiques.

S'y ajoutent des outils qui permettent aux élèves d'avancer à leur rythme, en étant autonomes dans leur travail, lors des séances de travail individuel ${ }^{16}$ pendant lesquelles ils remplissent leur contrat hebdomadaire. Le travail accompli est ensuite évalué avec l'enseignant, ce qui permet à l'élève de mieux se connaître et d'ajuster ses comportements aux apprentissages. Lorsqu'il y a des difficultés, il bénéficie de soutien personnalisé ou en groupe de besoin, au sein duquel il travaille sur les mêmes connaissances que ses camarades mais à des rythmes différents, avec des outils différents.

Lors des ateliers décloisonnés, les enfants travaillent avec les autres classes et enseignants, ce qui leur permet d'être confrontés à des plus grands ou plus petits qu'eux. Partie intégrante du projet de l'école, ces ateliers, qui permettent de pratiquer l'interdisciplinarité, ont lieu tout au long de l'année, d'une façon hebdomadaire mais aussi ponctuellement selon les moments de l'année : lors du carnaval pour préparer le programme des festivités, le défilé, les instruments de musique, ou à l'atelier plantation qui permet aux maternelles de planter des graines de

12. Maria Montessori préconise que l'enseignant ne doit pas se substituer à l'enfant dans ses activités, Roger Cousinet voit dans le maître un guide.

13. Caleb Gattegno (1911-1988), mathématicien, docteur ès lettres, crée la lecture en couleurs en 1957 alors qu'il est chargé d'une mission d'alphabétisation par l'UNESCO en Éthiopie.

14. Le matériel de la lecture en couleurs comprend le tableau de rectangles colorés (chaque rectangle correspond à un phonème), le pointeur (utilisé pour lier les graphèmes et en faire des mots, puis des phrases), le fidel (ensemble de huit tableaux où sont regroupés tous les graphèmes représentant un même phonème), les tableaux de mots, les cahiers d'exercice dans lesquels les élèves effectuent par écrit un travail personnel. 15. G. Cuisenaire (1891-1975), instituteur belge, invente les réglettes (matériel formé de petits morceaux de bois ou de plastique qui mesurent entre un et dix centimètres de longueur, d'une section de $1 \mathrm{~cm}^{2}$ et de couleurs différentes) dont C. Gattegno, qu'il rencontre en 1954, développe les possibilités.

16. Trois séances de 45 minutes par semaine. 
plantes aromatiques avec les CM1, d'observer les étapes de la croissance et les besoins des plantes, puis de les cuisiner lors de la confection de biscuits...

Quant à l'apprentissage du partage et de l'entraide, il se fait à différentes occasions, au quotidien lors du déjeuner apporté de la maison par chacun, ou à travers les différents métiers adaptés à la classe (le calendrier, le ménage, facteur, effaceur de tableau...) dont les enfants sont responsables, ou encore ponctuellement en participant à des associations à but humanitaire.

Les échanges avec l'extérieur se traduisent par des rencontres, enquêtes et sorties variées. Au quotidien, ils se retrouvent dans les correspondances avec d'autres écoles, et lors de l'apprentissage de l'anglais, fenêtre ouverte sur une autre culture. Deux enseignantes anglophones, l'une anglaise, l'autre américaine, se partagent les classes en demi-groupes, et leur apprennent les jeux, comptines, lectures, de leur pays. Elles participent à la vie de l'école au même titre que les autres enseignants et sont présentes lors des repas, des sorties, des classes transplantées, qui vont de trois jours à la ferme pour les plus petits à trois semaines aux États-Unis pour les plus grands. Les enfants anglophones, qui représentent un tiers de l'effectif, bénéficient du soutien de parents anglophones volontaires lorsque l'anglais écrit est abordé. Les parents, présents lors des nombreuses sorties pour lesquelles ils sont sollicités, viennent aussi à l'école pour partager leurs connaissances dans un domaine particulier, artistique, sportif ou autre.

\section{Les limites...}

Certaines sont incontournables : les frais de scolarité tout d'abord, qui imposent une barrière sociale dès l'entrée. La taille de l'école ensuite, qui permet à chacun des enseignants de connaître chaque enfant de l'école et d'établir un climat que l'on peut qualifier de familial, difficilement transposable. L'école garderait-elle son identité à plus grande échelle si sa philosophie, sa pédagogie, étaient appliquées dans des classes de trente enfants au lieu de vingt, avec une équipe d'enseignants plus élargie et surtout imposée ? Rien n'est moins sûr.

D'autres posent question. Que se passe-t-il lorsqu'un enfant, ou un parent, ne s'épanouit pas dans cet environnement ? Pression de la société par rapport à l'apprentissage des fondamentaux d'une part, prise en compte de l'épanouissement de son enfant au quotidien d'autre part, traduisent la tension à laquelle peuvent être soumises les familles. La position de l'équipe pédagogique sur ce point est très claire, dès lors que les dispositifs mis en place (rendez-vous parents-enseignants, travail avec l'éducatrice de jeunes enfants, médiation...) n'apportent pas de solution, il y a rupture de contrat, du côté de l'école ou de celui des parents, l'adhésion autour des principes inscrits dans la charte de l'école ne se faisant plus. La rupture est d'autant plus douloureuse que l'affectif y a sa part.

Une autre question se pose avec l'entrée au collège. Peu d'enfants poursuivent leur scolarité dans le circuit des écoles nouvelles ${ }^{17}$, ils rejoignent pour la plupart le système traditionnel, ce dernier restant la norme lors de l'entrée en sixième. Pour les parents ayant fait le choix d'une école différente, la confrontation à une

17. En région parisienne, l'école nouvelle La source, qui accueille les enfants de la maternelle au lycée, compte en 2010 seulement trois enfants de l'École aujourd'hui dans ses classes de lycée ; au sein de l'ANEN, seule l'école de la Prairie poursuit son expérience d'école nouvelle au collège 
réalité autre se traduit par une certaine peur de la stigmatisation. Les enfants quant à eux semblent plus sereins, " en bref, la sixième est un fossé plein de surprises, de mauvaises comme de bonnes. Il faut juste avoir confiance en soi », et «c'est moins difficile que ce qu'en disent les parents ${ }^{18}$ !».

Enfin, une limite inévitable tient au contrat qui lie l'école à l'État, induisant une certaine obéissance, sous peine de voir les subventions disparaître... Il s'agit alors de trouver les moyens de contourner les injonctions lorsqu'elles sont en conflit avec les principes de l'école, comme ce fut le cas lors des évaluations de CM2 en 2009, décriées au-delà de l'École aujourd'hui par une grande majorité d'enseignants. La liberté pédagogique ainsi s'arrête quelque part...

\section{Et demain?}

Il n'en reste pas moins que la communauté éducative rencontrée à l'École aujourd'hui-School for today interpelle par l'impression de bonheur qu'elle dégage, faisant fi de ce qui est le plus souvent montré du doigt par les médias... Non seulement les enfants y sont heureux, mais les adultes aussi ! Une utopie qui semble bien réelle, et à laquelle il fait bon croire... D'autant plus que l'adaptation à un enseignement traditionnel ne semble pas plus difficile pour les élèves d'une école dite à pédagogie nouvelle, comme le montre Rébecca Shankland dans sa thèse sur l'adaptation des étudiants. Après s'être demandée si les pédagogies nouvelles sont une aide à l'adaptation ou un facteur de marginalisation, elle propose des résultats en faveur d'une meilleure adaptation des élèves issus de ces écoles, se traduisant par confiance en soi, estime de soi et créativité (Shankland, 2009). C'est ce que semblent confirmer certains anciens parents de l'École aujourd'hui, soucieux dans un premier temps de la bonne intégration de leur enfant lors de l'entrée en sixième, puis rassurés de les voir se prendre en main, ils sont fiers de s'apercevoir qu'ils arrivent à concilier compétition et coopération, comme le fait remarquer ce parent dont le fils, dans une filière particulièrement compétitive, s'est investi dans le tutorat afin d'aider ses camarades de promotion. D'anciens élèves de l'école, parents d'élèves aujourd'hui, attribuent à la pédagogie dont ils ont bénéficié ici d'avoir acquis suffisamment de confiance en eux pour s'accrocher à leurs rêves contre vents et marées.

Les grands principes de l'Éducation nouvelle auraient-ils encore de beaux jours devant eux ? Il semblerait que cela soit d'autant plus vrai aujourd'hui que l'école traditionnelle laisse de moins en moins de place à l'enfant à l'école, tenu d'endosser son métier d'élève dès le plus jeune âge (Perrenoud, 1994).

Les expériences d'écoles innovantes, pour peu qu'elles se fassent connaître, pourraient bien avoir un bel avenir devant elles...

\section{Bibliographie}

BLOCh, M.-A. 1973. Philosophie de l'éducation nouvelle, Paris, PUf, $3^{\mathrm{e}}$ éd.

Fotinos, G. ; Testu, F. 1996. Aménager le temps scolaire : théories et pratiques, Paris, Hachette Éducation.

Montessori, M. 2004. L'enfant, Paris, Desclée de Brouwer.

Perrenoud, P. 1994. Métier d'élève et sens du travail scolaire, Paris, ESF Éditeur.

18. Cassia et Pierre-Louis, Arc-en-ciel n 35, juin 2009. 
SHankland, R. 2009. Pédagogies nouvelles et compétences psychosociales : de l'apprentissage à l'école à l'entrée dans l'enseignement supérieur, Paris, L’Harmattan.

"École aujourd'hui - School for today », Arc-en-ciel, numéro spécial 30 ans, 2005-2006, n 35, juin 2009.

\section{Outils mathématiques}

Les réglettes Cuisenaire, constituées de petits morceaux de bois ou de plastique longues de un à dix centimètres, d'une section de $1 \mathrm{~cm}^{2}$ et de couleurs différentes, permettent aux élèves, à travers leur manipulation, d'avoir une vision concrète des opérations mathématiques.

Les mosaïques Attrimaths, composées de blocs de formes et de couleurs différentes, permettent de développer les notions des mathématiques et de la géométrie.

Les Géoplans, grâce à des fiches utilisées avec des élastiques et un support à pointes, permettent de tracer des formes géométriques et de développer la structuration spatiale, la symétrie, etc.

Les TOP, outil Freinet, sont des cahiers de techniques opératoires visant à acquérir le calcul mental, les élèves faisant les exercices chacun à leur rythme.

Picbille, petit personnage spécialiste des billes de la méthode "J'apprends les maths » de Rémi Brissiaud, va aider les enfants à faire leurs premiers pas dans le calcul, en allant de la manipulation à la représentation d'images mentales. 


\section{Another Brick in the Wall}

We don't need no education

We don't need no thought control

No dark sarcasm in the classroom

Teachers leave them kids alone

Hey! Teacher! Leave the kids alone!

All in all it's just another brick in the wall.

All in all you're just another brick in the wall.

\section{Une brique de plus dans le mur}

Nous n'avons pas besoin d'éducation

Nous n'avons pas besoin de contrôle des pensées.

Pas de sombre sarcasme en classe.

Professeurs, laissez les enfants tranquilles

Eh! Le prof! Fiche la paix aux gamins!

De toute façon, ce n'est qu'une brique de plus dans le mur

De toute façon, vous n'êtes qu'une brique de plus dans le mur

Pink Floyd (Roger Waters)

En 1980, en Afrique du sud, cette chanson contestataire, qui dénonce la rigidité des règles scolaires en général et celle des internats en particulier, fut adoptée comme hymne contestataire par des étudiants noirs protestant contre l'apartheid qui sévissait alors dans les écoles du pays, et fut par conséquent officiellement interdite par le gouvernement sud-africain.

Dans la version du film The Wall et dans le clip vidéo, la chanson suit une séquence où les enfants marchent à travers des machines qui les formatent, les affublant de masques et d'uniformes absolument identiques, pour finalement entrer dans un hachoir à viande. À la fin, les enfants se révoltent et détruisent l'école. On comprend qu'on est dans l'imaginaire de l'élève Pink, blessé que son professeur l'ait ridiculisé en lisant un de ses poèmes devant le reste de la classe. 